\title{
Surgical treatment of intracardiac leiomyomatosis
}

\author{
Hui-Li Gan, MD, PhD, Jian-Qun Zhang, MD, Qi-Wen Zhou, MD, Qing-Yu Kong, MD, \\ Shuang Zhao, MD, and Ping Bo, MD
}

Objectives: Confusion exists regarding surgical algorithms for treating intracardiac leiomyomatosis. This report outlines the surgical management and outcomes of patients with intracardiac leiomyomatosis.

\begin{abstract}
Methods: Sixteen cases of intracardiac leiomyomatosis surgically treated in Anzhen Hospital from February 1995 to July 2010 were reviewed retrospectively. According to relative size and location of intracardiac leiomyoma maximum diameter relative to diameter of inferior vena cava, the 16 cases were classified as type A, $\mathrm{B}, \mathrm{C}$, or D.
\end{abstract}

Results: Of the 16 cases in this series, there were 7 type A, 2 type B, 3 type C, and 4 type D. No patients died during surgery. Mean follow-up was $90 \pm 57.1$ months (cumulative, 120.2 patient-years; range, 2-190 months). One patient died of recurrence 5 months after the surgery because of incomplete resection. Another patient with type $\mathrm{D}$ also died of recurrence 2 years after the primary procedure. A patient with type $\mathrm{D}$ died suddenly 10 years after the primary procedure. The 5-year and 10-year survivals calculated by the Kaplan-Meier method were $87.1 \% \pm 8.6 \%$ and $72.5 \% \pm 15 \%$. Of the 13 surviving patients, 11 were in New York Heart Association functional class I and 2 were in functional class II.

Conclusions: Surgical treatment of intracardiac leiomyomatosis can result in satisfactory midterm to long-term survival and satisfactory heart function. Multiple surgical strategies should be tailored to the anatomic characteristics of the intracardiac leiomyoma. Recurrence of intracardiac leiomyomatosis after the resection procedure may result in unfavorable late result (J Thorac Cardiovasc Surg 2011;142:823-8)

Intravenous leiomyoma (IVL) is an uncommon benign tumor that originates from the smooth muscle cells and is usually confined to the pelvic venous system. ${ }^{1}$ Intracardiac leiomyomatosis (ICL) occurs when an IVL extends into the right chambers of the heart through the inferior vena cava (IVC). Only about 300 cases of ICL have been described in the literature in single-case or case series reports. Because of its cardiac involvement, which may be deep into the pulmonary artery branches, ${ }^{2-4}$ and the complexity of its clinical presentations, ${ }^{5}$ much attention should be paid to the diagnosis and treatment of ICL. Here we report a cohort of 16 cases of ICL originating from the uterus and extending into the right atrium, right ventricle, or pulmonary artery.

\section{MATERIALS AND METHODS}

The ethics committee of Anzhen Hospital approved this retrospective study and waived the need to obtain patient consent for the study. Written,

\footnotetext{
From the Department of Cardiac Surgery, Beijing Anzhen Hospital, Capital Medical University, Beijing Institute of Heart, Lung and Blood Vessel Diseases, Beijing, China.

Supported by a grant (No. 81070041) from National Nature Science Foundation of China.

Disclosures: Authors have nothing to disclose with regard to commercial support.

Received for publication Sept 7, 2010; revisions received Dec 17, 2010; accepted for publication Jan 12, 2011; available ahead of print Feb 17, 2011.

Address for reprints: Hui-Li Gan, MD, PhD, Cardiac Surgery Department, Beijing Anzhen Hospital, Capital Medical University, Beijing Institute of Heart, Lung and Blood Vessel Diseases, Beijing 100029, China (E-mail: ganhuili@hotmail. com).

$0022-5223 / \$ 36.00$

Copyright (c) 2011 by The American Association for Thoracic Surgery

doi:10.1016/j.jtcvs.2011.01.023
}

informed consent had been obtained from each patient before their surgical treatment.

\section{Patient Characteristics}

From February 1995 to July 2010, a total of 16 patients with ICL, all of whom were female with ages ranging from 20 to 62 years (mean $44.8 \pm 9.6$ years), were operated on at Anzhen Hospital with multiple surgical strategies. The disease course ranged from 3 months to 2 years. Three patients were in New York Heart Association (NYHA) functional class IV, 11 were in class III, and the other 2 were in class II. Computed tomographic (CT)/magnetic resonance imaging scans, preprocedural transthoracic echocardiography, and abdominal color Doppler imaging were used to identify the site of origin and to assess the extent of vascular involvement for patients with ICL. As shown in Table 1 and Figure 1, the relative size and location of the intracardiac leiomyoma (maximum diameter relative to the diameter of the IVC) were used for retrospective classification of the lesions as type $\mathrm{A}, \mathrm{B}, \mathrm{C}$, or D. Of the 16 cases of ICL in this series, there were 7 type A, 2 type B, 3 type C, and 4 type D. The patients' characteristics are presented in Table 2. Echocardiography, CT scan, and abdominal color Doppler were used for the preoperative evaluation. The correct diagnosis of ICL was made preoperatively in 13 cases. The other 3 cases were misdiagnosed as right atrial myxoma or right atrial neoplasm, and the correct diagnosis was made during the excision procedure.

\section{Operative Procedures}

All 16 patients with ICL were operated on under general anesthesia. The surgical algorithms for the 16 cases of ICL are presented in Table 3. The 7 patients with type A ICL in were all approached through a short extra peritoneal incision in the lateral lower quadrant of the abdomen, and the internal iliac veins were dissected. Then the pedicles of the tumors were excised, and the tumors were taken out through internal iliac venotomy. The 2 patients with type B ICL were approached through sternotomy, cardiopulmonary bypass (CPB) was established through cannulation of the superior vena cava (SVC) and IVC, and the whole body of the intracardiac 


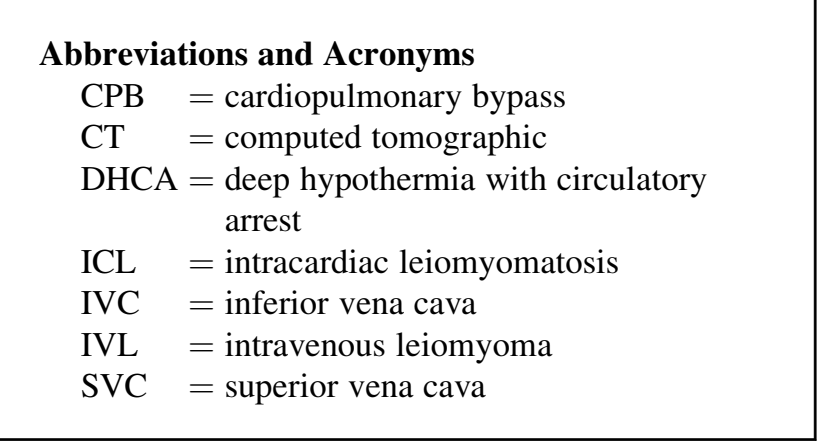

leiomyoma was taken out through the right atrial opening. One patient with type C ICL (patient 10) was approached through sternotomy, but the IVC could not be cannulated for establishment of CPB because of the blockage of the IVC by the tumor. Therefore deep hypothermia with low flow was applied through a single cannula in the SVC, and the intracardiac leiomyoma dilatation in the suprahepatic segment of the IVC was only partially excised. Another 2 patients with type C ICL were approached through sternolaparotomy, CPB was established, deep hypothermia with circulatory arrest (DHCA) was applied after dissection of the IVC, and then the tumor dilatation of the intracardiac leiomyoma and its 2 ends were excised through IVC venotomy. The 4 patients with type D ICL were approached through sternolaparotomy, CPB was established, DHCA was applied after dissection of the IVC, and then the intracardiac dilation of the tumor was taken out through the right atrial opening while the intracaval dilation was taken out through the IVC venotomy.

\section{Follow-up}

Follow-up at yearly intervals after the intracardiac leiomyoma excision procedure was completed for all 16 patients with ICL in the hospital outpatient departments with CT scan, echocardiography, and abdominal color Doppler. Mean follow-up was $90 \pm 57.1$ months (cumulative, 120.2 patient-years; range, 2-190 months).

\section{Statistical Analyses}

All statistical analyses were performed with the statistical software package SPSS 17.0 (SPSS Inc, an IBM Company, Chicago, Ill). Categoric data are given as total numbers. Continuous data are given as mean $\pm \mathrm{SD}$. Survivals at 5 years and 10 years were calculated with the Kaplan-Meier survival curve and are expressed as mean $\pm \mathrm{SD}$.

\section{RESULTS}

For patients 8 to $16, \mathrm{CPB}$ duration ranged from 35 to 149 minutes (mean, $82.2 \pm 46.7$ minutes), and crossclamp time ranged from 0 to 78 minutes ( $43.6 \pm 39.2$ minutes). DHCA duration ranged from 26 to 36 minutes. Nasopharyngeal temperature during $\mathrm{CPB}$ was $28.7^{\circ} \mathrm{C} \pm 6.9^{\circ} \mathrm{C}$. All $\mathrm{CPB}$ was discontinued without incident, and postoperative courses were not complicated except for that of 1 patient who underwent a reoperative procedure because of bleeding. All patients recovered well from the surgery. The blood loss was $530.5 \pm 246.7 \mathrm{~mL}$, and the transfusion requirement was $446.4 \pm 235.7 \mathrm{~mL}$. The intensive care unit stay was 24 \pm 11.2 hours, and the postoperative hospital stay was 11.3 \pm 5.2 days. In all 16 cases, the pathologic findings were extensive, hard, rubbery lesions, most of them yellowish or
TABLE 1. Classification of intracardiac leiomyomatosis

\begin{tabular}{|c|c|}
\hline Type & Description \\
\hline A & $\begin{array}{l}\text { The maximum diameters of both intracardiac and intracaval } \\
\text { sections of the tumor are smaller than the minimum } \\
\text { diameter of the IVC. }\end{array}$ \\
\hline B & $\begin{array}{l}\text { Only the maximum diameter of the intracardiac section of } \\
\text { ICL is bigger than the minimum diameter of the IVC. }\end{array}$ \\
\hline $\mathrm{C}$ & $\begin{array}{l}\text { Only the maximum diameter of the intracaval section } \\
\text { of ICL is bigger than the minimum diameter of the IVC. }\end{array}$ \\
\hline $\mathrm{D}$ & $\begin{array}{l}\text { The maximum diameters of both intracardiac and intracaval } \\
\text { sections of ICL are bigger than the minimum } \\
\text { diameter of the IVC. }\end{array}$ \\
\hline
\end{tabular}

reddish polymorphic tumors. Histologic analysis demonstrated these to be leiomyomas, and some with grossly dilated IVC suggested a protracted clinical course. The intravascular lesions measured 30 to $48 \mathrm{~cm}$ in length and 0.5 to $7.2 \mathrm{~cm}$ in width at the widest dilation. Positive estrogen receptors were detected in 5 cases.

Of the 16 patients, 8 had undergone hysterectomy or subtotal hysterectomy (as shown in Table 2) before the intracardiac leiomyoma excision procedure. Of the remaining 8 patients, 1 underwent a salpingo-oophorectomy during the primary procedure and 4 underwent second-stage salpingo-oophorectomy 2 to 4 weeks after the primary procedure. Another 2 patients underwent resection of pelvic fibroids 4 weeks after the primary procedure. For 15 patients, the internal iliac veins or the ovarian veins from which the intracardiac leiomyoma originated were ligated after the primary intracardiac leiomyoma excision procedure. Another patient with type D (patient 15) refused to undergo second-stage hysterectomy or subtotal hysterectomy and ligation of the originating vein after the primary procedure.

During the follow-up, 1 patient with type C ICL (patient 10) had a recurrence with a full blockage of the IVC in the third month after the primary procedure because of the incomplete excision of the lesion. The patient refused a second operation and died of pulmonary embolization 2 months later. Another patient with type D ICL (patient 15) also died of recurrence of the tumor 2 years after the primary procedure. A patient with type D ICL (patient 13) died suddenly 10 years after the primary procedure. As shown in Figure 2, the 5-year and 10-year survivals calculated with the Kaplan-Meier method were $87.1 \% \pm 8.6 \%$ and $72.5 \% \pm 15 \%$. Of the 13 surviving patients, 11 were in NYHA functional class I and 2 were in NYHA functional class II.

\section{DISCUSSION}

\section{The General Image of ICL}

ICL was first reported in 1907 from an autopsy analysis. ${ }^{6}$ All reported patients were women, ranging in age from 26 to 

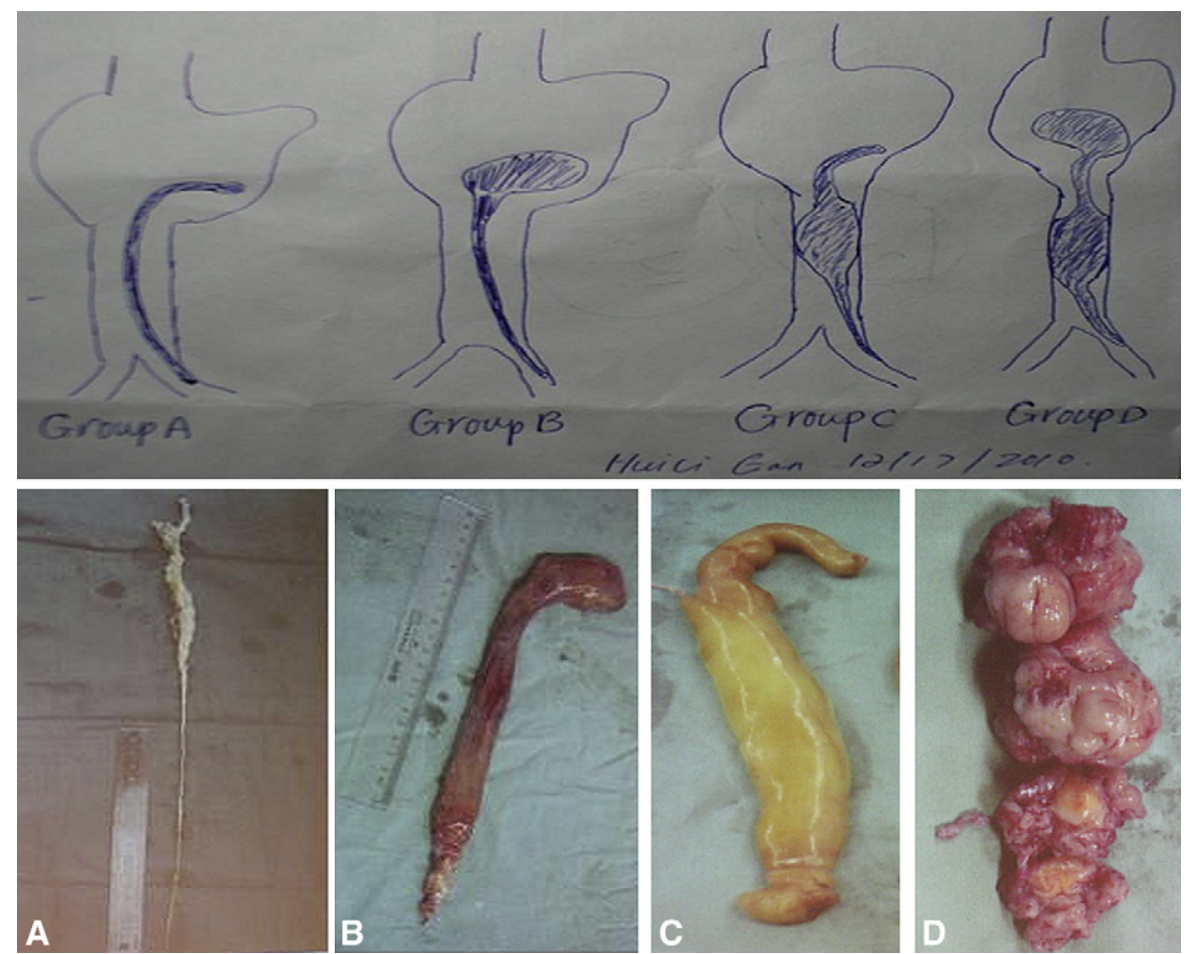

FIGURE 1. Photo shows schematic diagrams (upper panel) and pathologic specimens (lower panel) for types A, B, C, and D of intracardiac leiomyomatosis.

72 years and with a median age of 47 years. ${ }^{7}$ Patients sometimes do not have symptoms. ${ }^{8}$ IVL most commonly enters through the lumen of the iliac vein or ovarian vein, grows into the IVC, and sometimes reaches the right atrium, ventricle, and pulmonary artery. ${ }^{5,9}$ The tumor has always been found attached to the vascular bed of the internal iliac vein or the ovarian vein. Research on the histogenesis of ICL has revealed that the intracardiac leiomyoma does not originate from the vessel wall but rather from the seeding of the uterus and its adnexa. ${ }^{10}$
Although leiomyoma is histologically benign and characterized by slow growth, ICL can lead to such severe complications as syncope, right heart failure, or ascites and edema in the lower extremities. Radical excision is therefore essential once ICL has been diagnosed. Incomplete excision will result in recurrences that require further surgery or lead to death, as in the case of patient 10 in our series.

Because of poor documentation of this unusual condition, there are no guidelines for reporting and surgical management of ICL. We classified the 16 cases of ICL in our

\section{TABLE 2. Patient characteristics in 16 cases of intracardiac leiomyomatosis and adjuvant procedures}

\begin{tabular}{|c|c|c|c|c|}
\hline No. & Type & Age (y) & Main symptoms & Adjuvant procedures \\
\hline 1 & A & 20 & Palpitation & Salpingo-oophorectomy, internal iliac vein ligation \\
\hline 2 & A & 42 & Palpitation & Salpingo-oophorectomy, internal iliac vein ligation \\
\hline 3 & A & 39 & Palpitation & Salpingo-oophorectomy, internal iliac vein ligation \\
\hline 4 & A & 62 & None & Salpingo-oophorectomy, ovarian vein ligation \\
\hline 5 & A & 40 & Palpitation & Pelvic fibroid resection, ovarian vein ligation \\
\hline 6 & A & 56 & Palpitation & Subtotal hysterectomy, internal iliac vein ligation \\
\hline 7 & A & 47 & None & Hysterectomy, internal iliac vein ligation \\
\hline 8 & B & 48 & Palpitation, hemoptysis & Hysterectomy, internal iliac vein ligation \\
\hline 9 & B & 45 & Palpitation, & Hysterectomy, ovarian vein ligation \\
\hline 10 & $\mathrm{C}$ & 44 & Ascites, edema, syncope & Hysterectomy, no ligation \\
\hline 11 & $\mathrm{C}$ & 37 & Ascites, edema, syncope & Pelvic fibroid resection, internal iliac vein ligation \\
\hline 12 & $\mathrm{C}$ & 52 & Ascites, edema, syncope & Hysterectomy, internal iliac vein ligation \\
\hline 13 & $\mathrm{D}$ & 39 & Palpitation, hemoptysis & Salpingo-oophorectomy, internal iliac vein ligation \\
\hline 14 & $\mathrm{D}$ & 41 & Palpitation, ascites, syncope & Subtotal hysterectomy, internal iliac vein ligation \\
\hline 15 & $\mathrm{D}$ & 44 & Palpitation, ascites, syncope & No adjuvant procedures \\
\hline 16 & $\mathrm{D}$ & 46 & Palpitation, ascites & Hysterectomy, internal iliac vein ligation \\
\hline
\end{tabular}

Edema refers to edema at lower extremities. 
TABLE 3. Classification and surgical algorithms for the 16 cases of intracardiac leiomyomatosis

\begin{tabular}{lclll}
\hline No. & Type & \multicolumn{1}{c}{ Approach } & CPB style & Cannulation \\
\hline 1 & A & Laparotomy & None & None \\
2 & A & Laparotomy & None & None \\
3 & A & Laparotomy & None & None \\
4 & A & Laparotomy & None & None \\
5 & A & Laparotomy & None & None \\
6 & A & Laparotomy & None & None \\
7 & A & Laparotomy & None & None \\
8 & B & Sternotomy & Moderate & SVC and IVC \\
& & & hypothermia & \\
& & & CPB & \\
9 & B & Sternotomy & Moderate & SVC and IVC \\
& & & hypothermia & \\
& & & CPB & \\
10 & C & Sternotomy & DHLF & SVC \\
11 & C & Sternolaparotomy & DHCA & SVC and IVC \\
12 & C & Sternolaparotomy & DHCA & SVC and IVC \\
13 & D & Sternolaparotomy & DHCA & SVC and IVC \\
14 & D & Sternolaparotomy & DHCA & SVC and IVC \\
15 & D & Sternolaparotomy & DHCA & SVC and IVC \\
16 & D & Sternolaparotomy & DHCA & SVC and IVC \\
\hline
\end{tabular}

$C P B$, Cardiopulmonary bypass; $S V C$, superior vena cava; $I V C$, inferior vena cava; $D H L F$, deep hypothermia and low flow; $D H C A$, deep hypothermia and circulatory arrest.

series into 4 different types (Table 1 ) according to the relative size and location of the intracardiac leiomyoma (maximum diameter vs the diameter of the IVC). This classification method can be used to categorize ICL cases into different types according to their anatomic characteristics, thus simplifying the description and reporting of future ICL cases. This classification method can also be used to stratify and understand the symptoms of different types of ICL. Occlusion of the IVC can lead to typical signs and symptoms of right heart failure. ${ }^{11}$ Total obstruction of the tricuspid valve will result in sudden death. ${ }^{12,13}$ This kind of emergency mostly appears in type B ICL, which has large tumor dilatation at its intracardiac free end. For type A ICL, however, there are no signs of lower extremity edema or ascites or incarceration in the right side chambers of the heart. Patients with type C or D ICL would have signs of IVC blockage, leading to collateral circulation established around the blockage, so that much more hemorrhaging would occur when dissecting the IVC during the resection procedure. ${ }^{8}$

\section{The Diagnosis of ICL}

CT scan provides the best diagnostic information, because it can identify the site and size of an intravenous tumor with or without the involvement of the right chambers of the heart. ${ }^{14,15}$ Echocardiography and abdominal color Doppler were also adopted for the preoperative evaluation. In 13 cases of ICL in our series, the correct diagnosis was made preoperatively, whereas in another 3 cases the correct

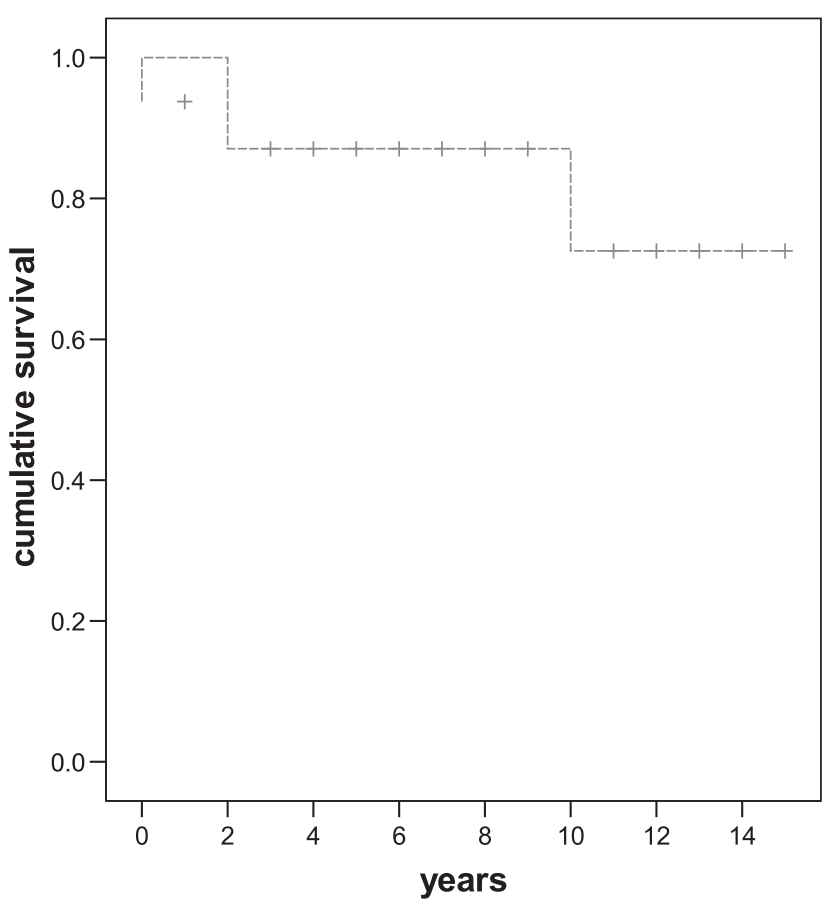

FIGURE 2. Kaplan-Meier curve for cumulative survival after intracardiac leiomyoma resection procedure.

diagnosis of ICL was not made preoperatively. Diagnosis before the operation can be made on the basis of echocardiography, CT angiographic scan, and abdominal color Doppler test. The use of venous-phase CT angiography, magnetic resonance angiography, transesophageal echocardiography, conventional venography, and intravascular ultrasonography, which could provide more details about the tumors, may further heighten the rate of correct diagnosis. Because of the relatively occult and severe clinical presentations of ICL, it is recommended that echocardiography, routine CT scan, and abdominal color Doppler be adopted during lifelong follow-up at yearly intervals to ensure early and timely diagnosis of any recurrence.

ICL should be considered in the differential diagnosis of a middle-aged woman with a right-sided heart tumor, especially for patients with previous hysterectomy or subtotal hysterectomy. ${ }^{16}$ The symptoms of ICL can range from insidious (type A) to syncope (type C or D) or even sudden death; its clinical manifestations are diverse but not specific, resulting in higher rates of misdiagnosis and missed diagnosis. Some patients, such as patient 13 in our series, may even reveal a symptom of hemoptysis, which may be a clue to pulmonary embolism caused either by the dropping of the intracardiac leiomyoma or by the blood embolism. In our series, 3 cases were misdiagnosed as right atrial myxoma or right atrial neoplasm. The reason for this misdiagnosis is that after the echocardiography identified tumors in the right heart system, no further attempt was taken to track the source of the tumors through CT 
angiographic scan. For the rare entity of ICL, correct diagnosis relies on a huge index of suspicion.

\section{The Surgical Strategy for ICL}

Intracardiac leiomyoma is a rare, benign neoplasm that usually originates from uterus and its adnexa and grows within the pelvic venous system and IVC. The tumor is sometimes characterized by a large dilation in its proximal end or its body but with a small distal originating end in the internal iliac vein or ovarian vein. The location of the dilation always determines the strategy of the surgical procedure. Although the intravascular part of the intracardiac leiomyoma is a kind of smooth muscle cell tumor that grows within venous channels and does not invade the tissues, and intracardiac leiomyomas are covered with smooth endometrial or endothelium, the tumor sometimes does adhere to the iliac vein, the IVC, or the right heart system when its diameter is larger than the diameter of the vein it courses. Thus these tumors cannot be taken out easily; only after meticulous and careful dissection to separate the tumors from the vein can such an intracardiac leiomyoma be taken out completely.

The most important purpose of our ICL classification method is to provide a general basis for surgical algorithms for management of ICL. Type A ICL has no obvious intracardiac or intracaval dilatation; therefore, it could be treated through a single pelvic approach, whereby the entire tumor can be retrieved through internal iliac or pelvic venotomy. CPB support and sternotomy could be avoided. ${ }^{17-19}$

In type B ICL, where the intracardiac section of the intracardiac leiomyoma is larger than the other segments, the tumor can be approached through sternotomy and the CPB can be established through cannulation of both the SVC and IVC. The intracaval and intracardiac sections of the intracardiac leiomyoma in type B ICL must be taken out through the right atrial opening. If danger from blind pulling is a concern, a short lateral lower abdominal incision could be added to excise the pedicle of the tumor in the internal iliac or ovarian vein. In this way, a long sternolaparotomy could be avoided.

Type C or D ICL should be approached through sternolaparotomy, and the CPB should be established through cannulation of the SVC and IVC. The dissection of the tumor should proceed under the support of DHCA, especially when the tumor dilation is located at the retrohepatic segment of the IVC.

Intracardiac leiomyoma attachment is usually located in the pelvic veins, and attempted removal by the thoracic approach would possibly result in either failure of complete retrieval of the tumor or tears in the vein at the site of attachment. Intraoperative deaths have occurred from massive retroperitoneal hemorrhage after avulsion of the tumor from the cardiac end, so for safety reasons we would recommend that the pedicle of the intracardiac leiomyoma be excised through a lateral lower abdominal incision. The recurrence of the IVL and ICL could be prevented through second-stage hysterectomy and ligation of the internal iliac vein or ovarian vein. ${ }^{19}$ Preprocedural or postoperative hysterectomy or salpingo-oophorectomy or ligation of the originating vein before or after the primary procedure is also very important for the prevention of the recurrence of the leiomyomatosis. One patient with type D ICL (patient 15), who did not undergo hysterectomy, salpingo-oophorectomy, or ligation of the originating vein after the primary procedure had recurrence and died 2 years later.

If the tumor is too extensive, or adheres to the cardiac and vascular structures, requiring resection of the abdominopelvic and intrathoracic components, a separate operation may be mandatory. ${ }^{20}$ Otherwise, single-stage resection under the support of DHCA could proceed with success. ${ }^{11,21-23}$ As a simple and safe approach to removing the iliocaval portion of the IVL, iliac venotomy could be used in 1- and 2-stage operations. ${ }^{24}$

Surgical mortality is low, and the follow-up study in our series suggests that the recurrence rate is also low. The only 2 recurrences in our series were related to the incomplete excision of the intracardiac leiomyoma in type C ICL or a lack of the ligation of the internal iliac vein or its tributary in type D ICL. All surviving patients had a satisfactory NYHA functional status after the excision procedure.

\section{Study Limitations}

This study is a retrospective analysis, and some confounding factors may blur the real image the early and late prognoses and the potential for recurrence after an intracardiac leiomyoma excision procedure. To define more exactly the efficacy of intracardiac leiomyoma excision procedure, further studies in a wider range are needed to confirm the findings of this study.

\section{CONCLUSIONS}

The surgical treatment of ICL can result in satisfactory midterm to long-term survival and satisfactory heart function. Multiple surgical strategies should be tailored according to the anatomic characteristics of the intracardiac leiomyoma. Recurrence of ICL after the resection procedure may be associated with an unfavorable late result.

\section{References}

1. Ma SQ, Bai CM, Yu XH, Huang OP, Lang JH, Li J. [Clinical and pathological analysis of intravenous leiomyomatosis]. Zhonghua Fu Chan Ke Za Zhi. 2005 40:34-7. Chinese.

2. Akatsuka N, Tokunaga K, Isshiki T, Asano K, Funaki H, Mizuno M, et al. Intravenous leiomyomatosis of the uterus with continuous extension into the pulmonary artery. Jpn Heart J. 1984;25:651-9.

3. Ricci MA, Cloutier LM, Mount S, Welander C, Leavitt BJ. Intravenous leiomyomatosis with intracardiac extension. Cardiovasc Surg. 1995;3:693-6.

4. Gawne-Cain ML, Wilson AG, Corbishley C, Keating V, Joseph AE. Case report: intravenous leiomyomatosis, an unusual cause of intracardiac filling defect. Clin Radiol. 1995;50:123-5. 
5. Wakiyama H, Sugimoto T, Ataka K, Yamashita C, Tsuji Y, Nakagiri K, et al. Intravenous leiomyomatosis extending into the right ventricular cavity: one-stage radical operation using cardiopulmonary bypass-a case report. Angiology. 2000;51:505-9.

6. Durck H. Ein kontinuierlich durch die untere. Hohlvene in das Herz vorwaschsendes Leiomyom. München Med Wochnschr. 1907;54:1154-5. German.

7. Lam PM, Lo KW, Yu MY, Wong WS, Lau JY, Arifi AA, et al. Intravenous leiomyomatosis: two cases with different routes of tumor extension. J Vasc Surg. 2004;39:465-9.

8. Topcuoglu MS, Yaliniz H, Poyrazoglu H, Tokcan A, Demir SC, Bozkurt A, et al. Intravenous leiomyomatosis extending into the right ventricle after subtotal hysterectomy. Ann Thorac Surg. 2004;78:330-2.

9. Mandelbaum I, Paulletto FJ, Nasser WK. Resection of a leiomyoma of the inferior vena cava that produced tricuspid valvular obstruction. J Thorac Cardiovasc Surg. 1974;67:561-7.

10. Kir G, Kir M, Gurbuz A, Karateke A, Aker F. Estrogen and progesterone expression of vessel walls with intravascular leiomyomatosis; discussion of histogenesis. Eur J Gynaecol Oncol. 2004;25:362-6.

11. Cooper MM, Guillem J, Dalton J, Marboe CC, Corwin S, Todd GJ, et al. Recurrent intravenous leiomyomatosis with cardiac extension. Ann Thorac Surg. 1992; 52:139-41.

12. Gonzalez-Lavin L, Lee HR, Falk L, Gradman MD, McFadden PM, Basso LV, et al. Tricuspid valve obstruction due to intravenous leiomyomatosis. Am Heart J. 1984;108:1544-6.

13. Rosenberg JM, Marvast MA, Obeid A, Johnson LW, Bonaventura M. Intravenous leiomyomatosis: a rare cause of right sided cardiac obstruction. Eur J Cardiothorac Surg. 1988;2:58-60.

14. Lai TK, Huang HY, Chan RY, Chin AC, Wong WC, Sit CY, et al. Magnetic resonance venogram of intravenous leiomyomatosis. Hong Kong Med J. 2005;11:524-6.
15. Wong YY, Chu WC, Lam WW. Intravenous leiomyomatosis: computed tomography diagnosis. Hong Kong Med J. 2006;12:239-40.

16. Mozzola A, Gregorini R, Procaccini B, Moretti V, Lucantoni K, Lorenzi W, et al. Intracaval and intracardiac leiomyomatosis of uterine origin. Ann Vasc Surg. $1986 ; 1: 134-8$

17. Moorjani N, Kuo J, Ashley S, Hughes G. Intravenous uterine leiomyosarcomatosis with intracardiac extension. J Card Surg. 2005;20:382-5.

18. Subramaniam B, Pawlowski J, Gross BA, Kim YB, LoGerfo FW. TEE-guided one-stage excision of intravenous leiomyomatosis with cardiac extension through an abdominal approach. J Cardiothorac Vasc Anesth. 2006;20:94-5.

19. Gehr NR, Lund O, Alstrup P. Recurrence of uterine intravenous leiomyomatosis with intracardiac extension. Diagnostic considerations and surgical removal. Recurrence of uterine intravenous leiomyomatosis with intracardiac extension. Diagnostic considerations and surgical removal. Scand Cardiovasc J. 1999;33: $312-4$.

20. Filsouri F, Farivar RS, Anderson C, Santerre D, Adams DH. Renal vein injury complicating removal of intravenous leiomyoma. J Thorac Cardiovasc Surg. 2002;123:820-2.

21. Okamoto H, Itoh T, Morita S, Matsuura A, Yasuura K. Intravenous leiomyomatosis extending into the right ventricle: one-stage radical excision during hypothermic circulatory arrest. Thorac Cardiovasc Surg. 1994;42:361-3.

22. Arinami Y, Kodama S, Kase H, Tanaka K, Okazaki H, Maruyama Y. Successful one-stage complete removal of an entire intravenous leiomyomatosis in the heart, vena cava, and uterus. Gynecol Oncol. 1997;64:547-50.

23. Katsumata T, Shinfeld A, Houel R, Westaby S. Pelvic leiomyoma in the right atrium. Ann Thorac Surg. 1998;66:2095-6.

24. Stolf NA, Santos G, Haddad VL, Simões RM, Avelar SF Jr, Ferreira FH Jr, et al. Successful one-stage resection of the intravenous leiomyomatosis of the uterus with extension into the heart. Cardiovasc Surg. 1999;7:661-4. 